\title{
INFLUENCE OF HOT ISOSTATIC PRESSING AND SURFACE FINISH ON THE MECHANICAL BEHAVIOUR OF TI6AL4V PROCESSED BY ELECTRON BEAM MELTING
}

\author{
Stefania Franchitti ${ }^{1}$, Carmine Pirozzi ${ }^{1}$, and Rosario Borrelli ${ }^{1}$ \\ ${ }^{1}$ Italian Aerospace Research Centre
}

April 28, 2020

\begin{abstract}
Electron Beam Melting (EBM) is one of a few additive manufacturing technologies capable of making full-density functional metallic parts realized from raw materials in the form of powders. The ability of direct fabrications of metallic parts can accelerate product designs and developments in a wide range of metallic-part applications, especially for complex components, which are difficult to make by conventional manufacturing means. To capitalize on these benefits, it must be shown that the mechanical performances of parts produced by EBM can meet design requirements. In this research an intensive mechanical characterization aimed at determining static and fatigue performance of the alloy Ti6Al4V processed by EBM has been performed. The effect of both postprocessing treatments (HIP and surface finish) on the mechanical behavior was evaluated by mechanical testing, microstructural study, computed tomography analysis and fracture surface investigation.
\end{abstract}

\section{Hosted file}

PAPER fatica_ULTIM013.docx available at https://authorea.com/users/309425/articles/440465-influenceof-hot-isostatic-pressing-and-surface-finish-on-the-mechanical-behaviour-of-ti6al4v-processedby-electron-beam-melting 

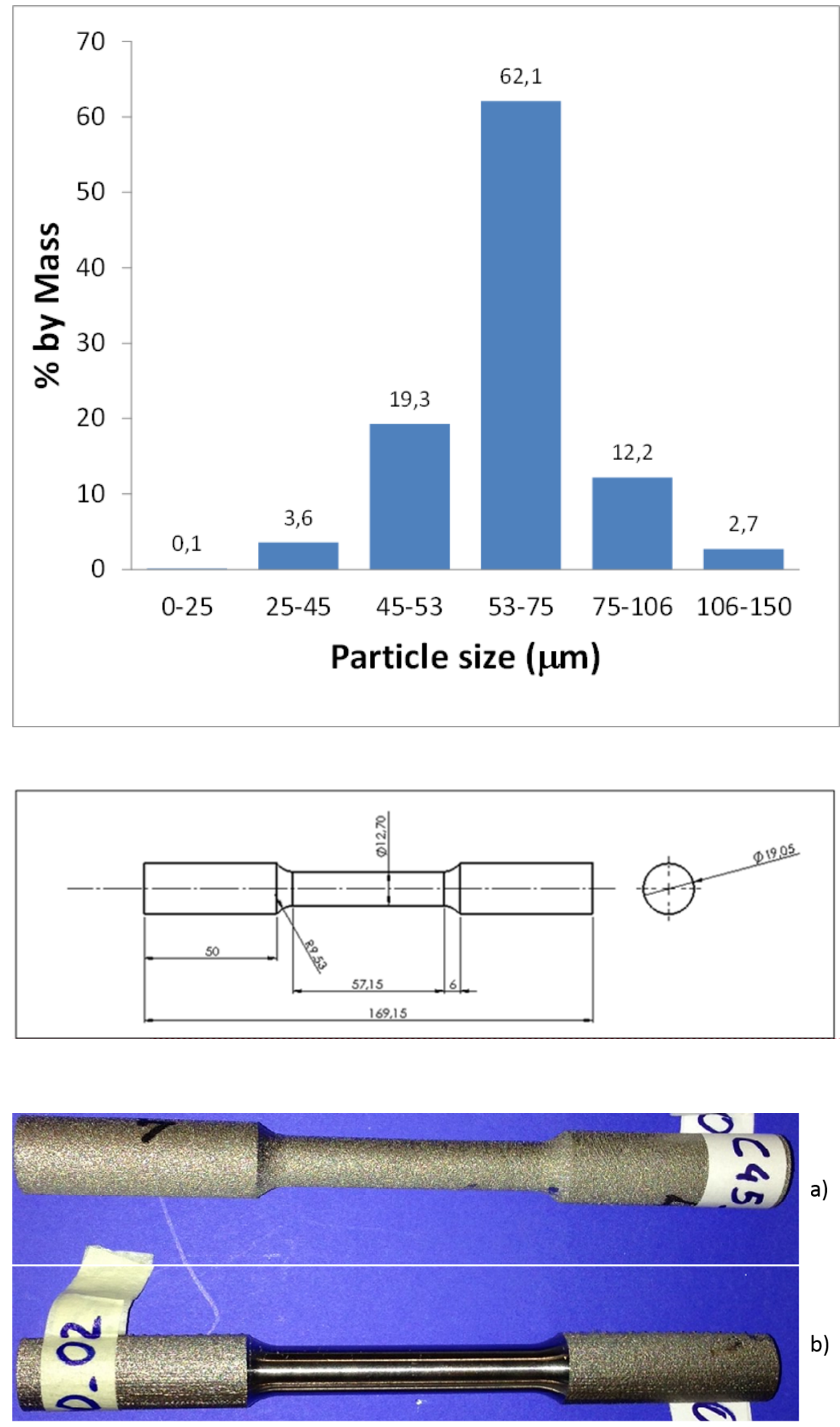

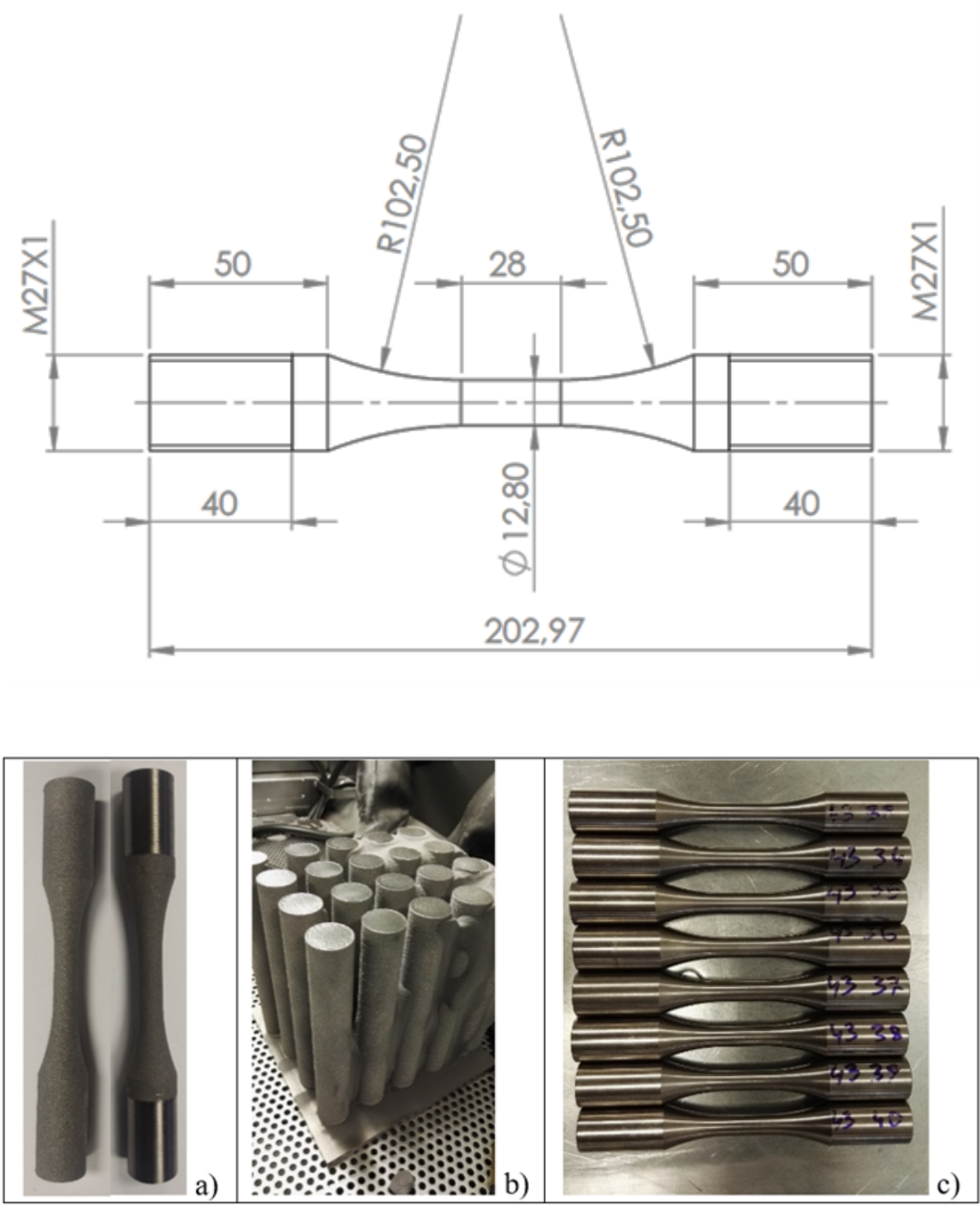

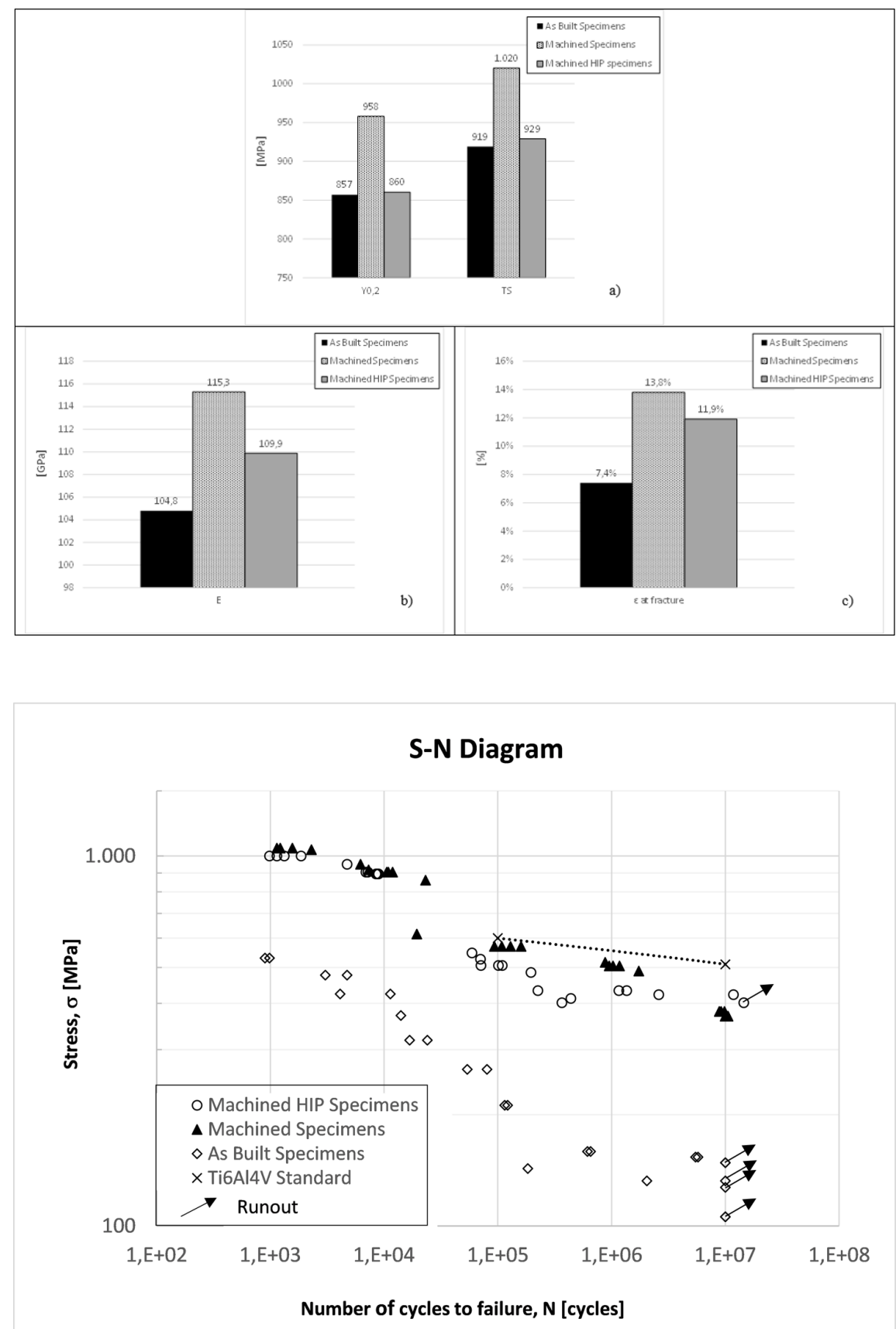
no HIP
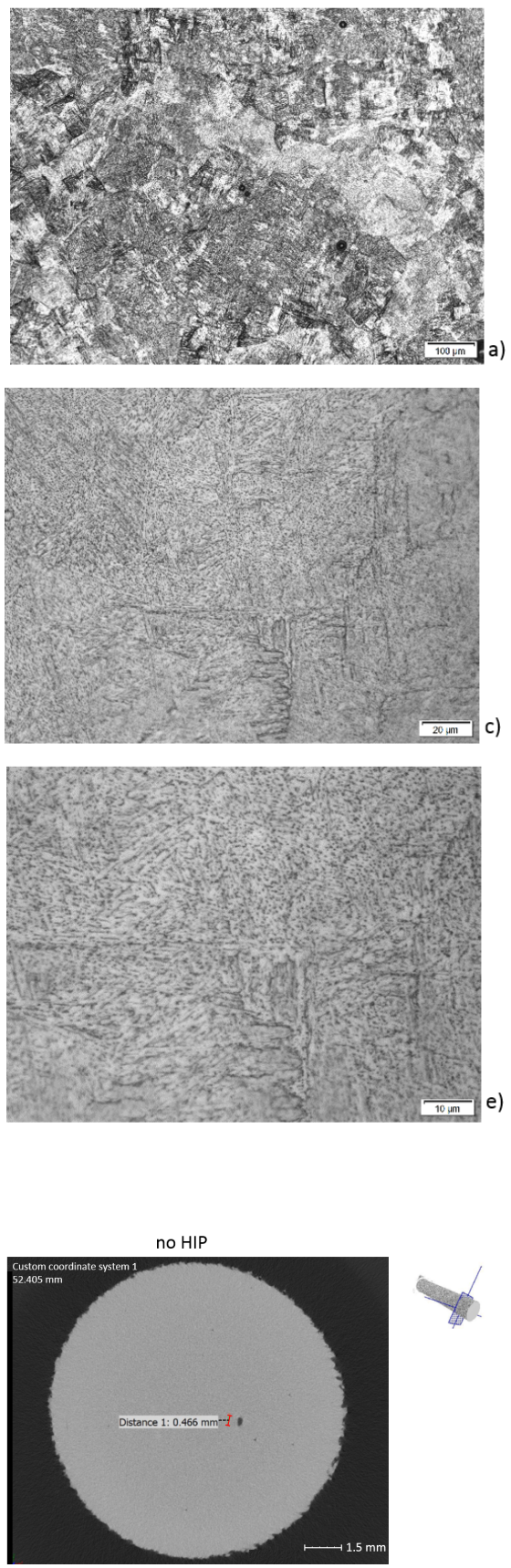

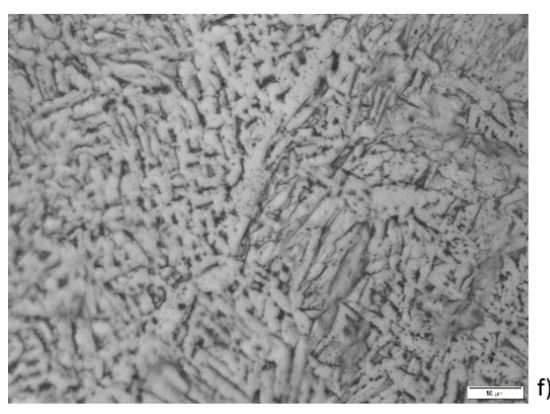

HIP
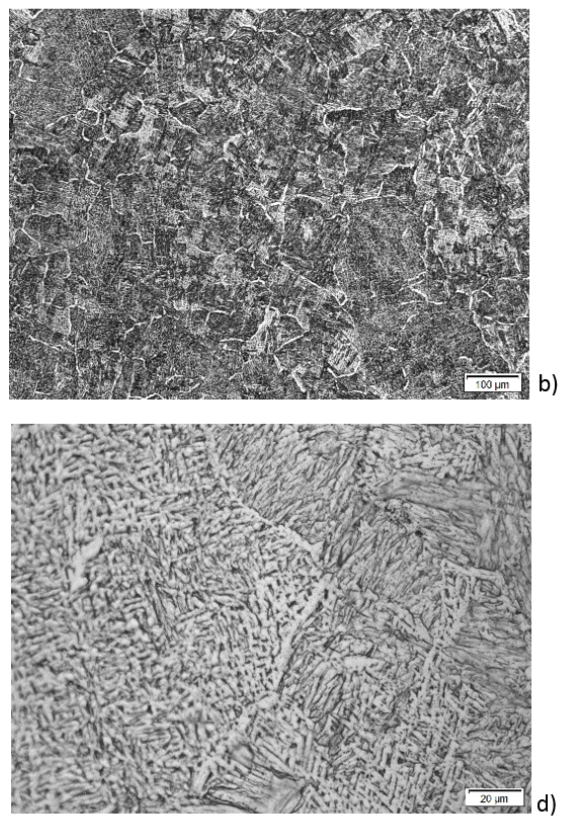

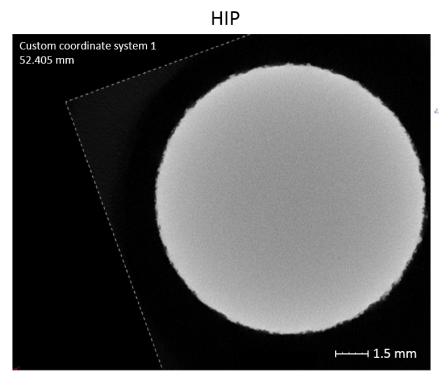




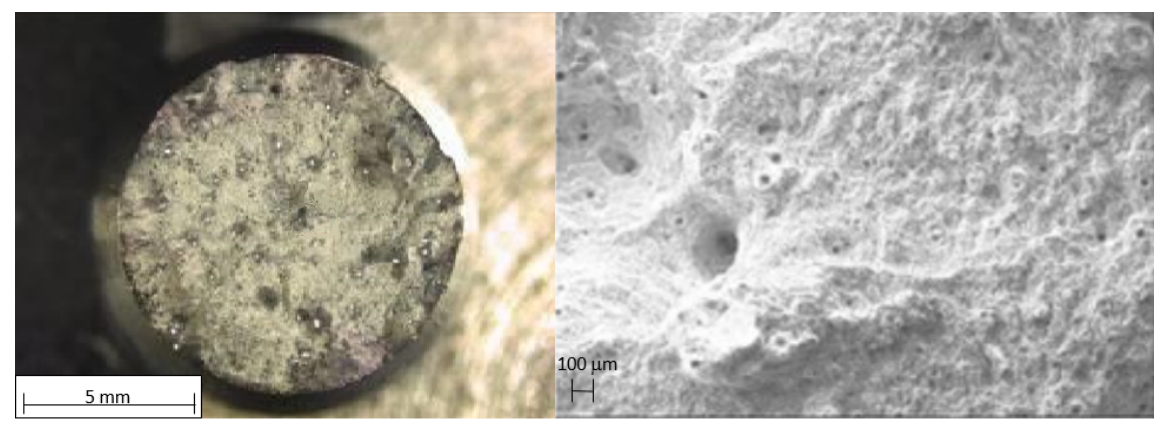

a)

b)

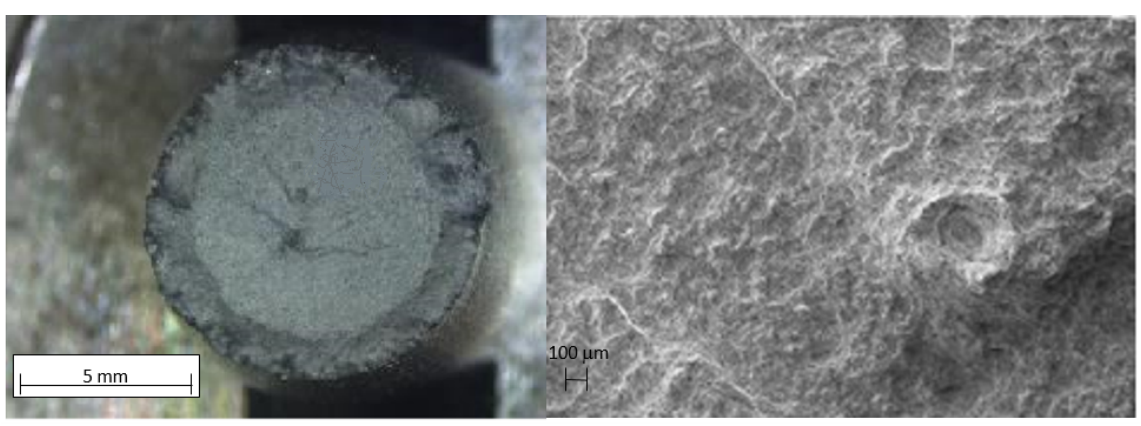

a)

b)

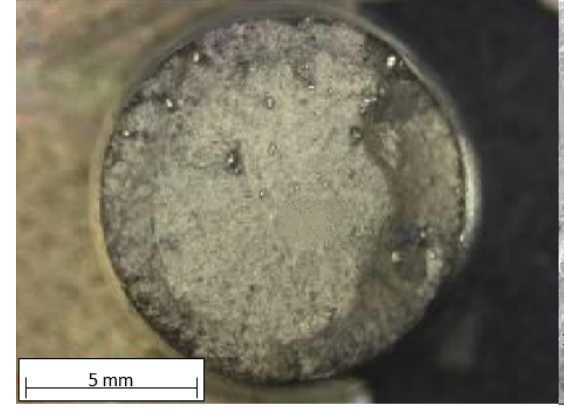

a)

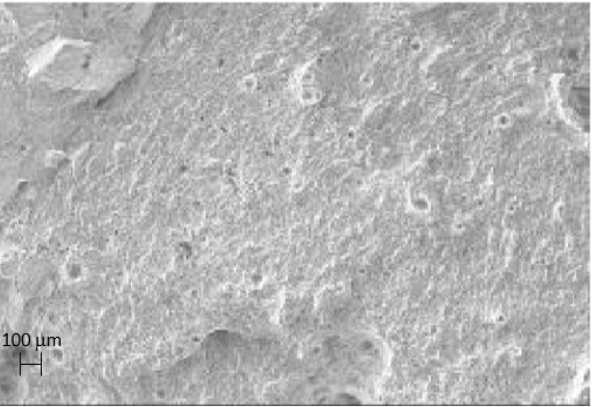

b)

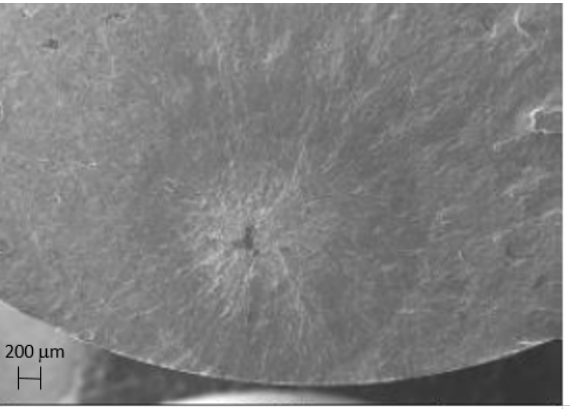

b) 


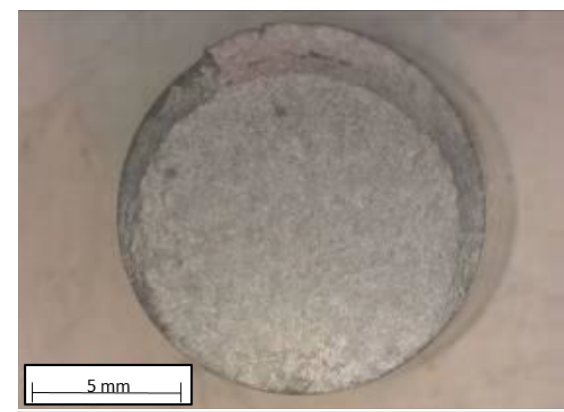

a)

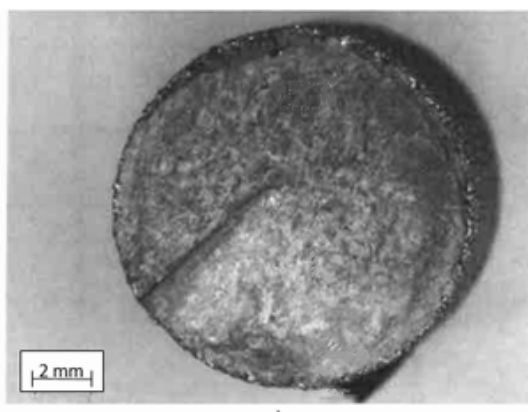

a)

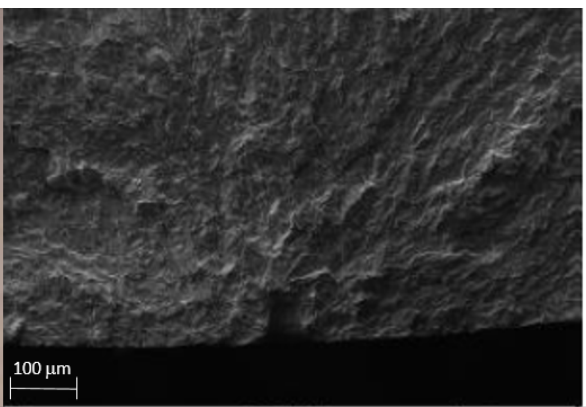

b)

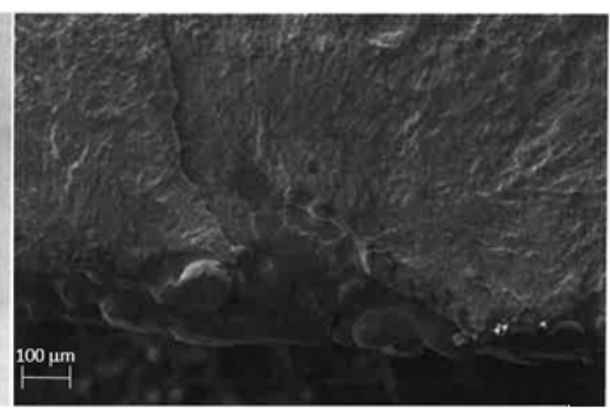

b)

\section{Hosted file}

table 1.docx available at https://authorea.com/users/309425/articles/440465-influence-of-hotisostatic-pressing-and-surface-finish-on-the-mechanical-behaviour-of-ti6al4v-processed-byelectron-beam-melting 